\title{
Low-frequency quantum transport in a three-probe mesoscopic conductor
}

\author{
Qingrong Zheng and Jian Wang \\ Department of Physics, The University of Hong Kong, Pokfulam Road, Hong Kong, China \\ Hong Guo \\ Centre for the Physics of Materials, Department of Physics, McGill University, Montreal, Quebec, Canada H3A 2T8
}

(Received 27 January 1997; revised manuscript received 23 June 1997)

\begin{abstract}
We investigate the low-frequency quantum transport properties of a three-probe mesoscopic conductor. The static transmission coefficients and emittance matrix of the system were computed by explicitly evaluating the various partial density of states (PDOS). We studied the finite-size effect of the scattering volume on the global PDOS. By increasing the scattering volume we observed a gradual improvement in the agreement of the total DOS as computed externally or locally. Our numerical data permit a particular fitting form of the finite-size effect. Finally, we propose a method to solve the finite-size problem. [S0163-1829(97)03043-9]
\end{abstract}

\section{INTRODUCTION}

The concept of partial density of states (PDOS) has been found to play an important role in the scattering approach to the low-frequency ac transport in coherent quantum conductors. ${ }^{1,2}$ For a scattering problem involving a multiprobe conductor, each scattering matrix element is associated with a PDOS which describes the density of states for transmission (or reflection). Furthermore, the low frequency admittance of a conductor is, in principle, calculable if the PDOS is known. ${ }^{1}$ Thus from the single-electron-scattering approach, the problem of predicting low-frequency admittance is reduced to the problem of finding the PDOS.

There are two kinds of PDOS which directly contribute to the admittance. ${ }^{1}$ First, the global PDOS (GPDOS) comes naturally from the external contribution to the electric current in a conductor when a carrier reservoir increases its electrochemical potential slightly. The scattering theory ${ }^{3}$ gives GPDOS to be

$$
\frac{d N_{\alpha \beta}}{d E}=\frac{1}{4 \pi i}\left(s_{\alpha \beta}^{\dagger} \frac{d s_{\alpha \beta}}{d E}-\frac{d s_{\alpha \beta}^{\dagger}}{d E} s_{\alpha \beta}\right),
$$

where $s_{\alpha \beta}$ is the scattering matrix element which connects a probe of the conductor labeled by $\beta$ to that by $\alpha$. Thus knowing $s_{\alpha \beta}$ as a function of the scattering electron energy $E$, one can compute GPDOS. The second kind of PDOS is the local PDOS (LPDOS), which arises in the scattering theory due to the present of a displacement current. In other words, the long-range Coulomb interaction of the charges gives rise to an internal response to the external perturbation, and this internal response is naturally expressed in terms of LPDOS. The scattering theory predicts ${ }^{1}$ LPDOS to be

$$
\frac{d n_{\alpha \beta}(\mathbf{r})}{d E}=-\frac{1}{4 \pi i} \operatorname{Tr}\left[\mathbf{s}_{\alpha \beta}^{\dagger} \frac{\delta \mathbf{s}_{\alpha \beta}}{e \delta U(\mathbf{r})}-\frac{\delta \mathbf{s}_{\alpha \beta}^{\dagger}}{e \delta U(\mathbf{r})} \mathbf{s}_{\alpha \beta}\right]
$$

Hence if we know the functional dependence of the scattering matrix on the scattering potential landscape $U(\mathbf{r})$, LPDOS can be obtained. Finally, the ac emittance matrix is calculable from these PDOS.
The PDOS possesses interesting properties. ${ }^{1}$ An important property which is physically reasonable is the relationship between the GPDOS and LPDOS,

$$
\frac{d N_{\alpha \beta}}{d E}=\int \frac{d n_{\alpha \beta}(\mathbf{r})}{d E} d^{3} \mathbf{r} .
$$

This expression relates a quantity which is computed at the scattering region boundary, $d N_{\alpha \beta} / d E$, to that evaluated within the scattering region. This equality also reflects the necessary condition of electrical current conservation which is a central requirement for low-frequency ac transport. In a previous work, ${ }^{4}$ using a simple but exactly solvable model, the authors have found that the equal sign of Eq. (3) is not always satisfied. The problem seems related to the fact that the left hand side of Eq. (3) is always evaluated at the boundary of a finite-size scattering region, hence there were finitesize corrections. The detailed understanding of these corrections, and more so the solution of this problem, are important issues in analyzing the low-frequency ac transport properties of mesoscopic conductors. Without the understanding and without a practical way of solving this finite-size problem, numerical calculation will likely produce results inconsistent with the current conservation requirement. Furthermore, since the low-frequency ac admittance comes from a linear combination of terms involving the GPDOS and the LPDOS, spurious results can be obtained due to the finite-size effect at certain energies (see below).

In this work, we shall focus on the finite-size problem of the GPDOS, and propose a practical solution of it. While the problem has been exposed in our exactly solved model, ${ }^{4}$ no practical numerical solution was yet known. Moreover, the exactly solved model is very special, being a two-probe quasi-one-dimensional (1D) wire with a point scatterer inside. This is not the typical mesoscopic multiprobe conductors fabricated or analyzed. In this work, we shall use a more general and typical three-probe system to examine the GPDOS, and use it to demonstrate a proposal of solving the finite-size problem. A spinoff of the work is to obtain the 


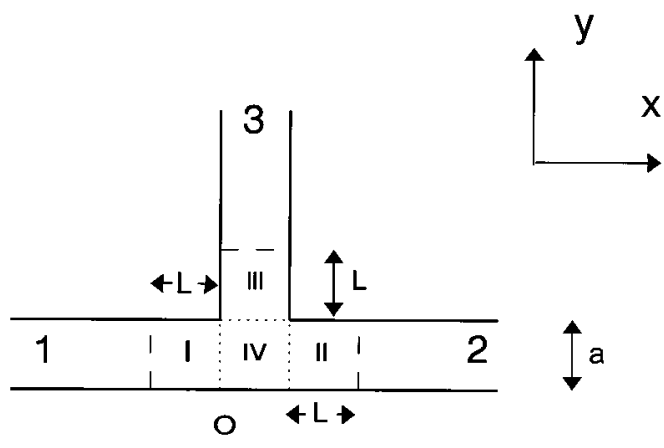

FIG. 1. Schematic plot of the three-probe quantum wire system. The scattering volume is defined by the dotted lines.

low-frequency ac admittance for the three-probe system: so far the only data in the literature for $2 \mathrm{D}$ conductors are for two-probe systems.

This paper is organized as follows. In the next section we present the numerical analysis of the GPDOS and LPDOS, focusing on the finite-size problem. In Sec. III we propose a practical method of solving the problem. Section IV presents the results for the low-frequency ac admittance. Finally, a summary is given in Sec. IV.

\section{FINITE-SIZE EFFECT TO THE GPDOS}

We analyze the three-probe system shown in Fig. 1 to illustrate the ac transport properties. For clarity we shall focus on the first transport subband by examining energies within the following range: $(\pi)^{2}<E<(2 \pi)^{2}$ in units of $\hbar^{2} /\left(2 m a^{2}\right)$ with $m$ the effective mass of the electron and $a$ the width of the leads [see Fig. 1]. Multiple subbands can be included without difficulties. The scattering properties of the three-probe system is then characterized by a $3 \times 3$ scattering matrix $\mathbf{S}(E) \equiv\left\{s_{\alpha \beta}\right\}$ with $\alpha, \beta=1,2,3$. The transmission coefficients can thus be expressed in terms of the scattering matrix, e.g., $T_{\alpha \beta}=\left|s_{\alpha \beta}\right|^{2}$. For the system of Fig. 1, the scattering matrix has the following symmetry: $\left|s_{11}\right|=\left|s_{22}\right|$, $\left|s_{21}\right|=\left|s_{12}\right|,\left|s_{31}\right|=\left|s_{32}\right|,\left|s_{13}\right|=\left|s_{23}\right|$, and $\left|s_{13}\right|=\left|s_{31}\right|$. Therefore, there are only four distinct elements out of nine.

We solve the quantum scattering problem using a mode matching method. The wave function in region I can be written as

$$
\Psi_{\mathrm{I}}=\sum_{n} \chi_{n}(y)\left(a_{n} e^{i k_{n} x}+b_{n} e^{-i k_{n} x}\right),
$$

where $\chi_{n}(y)$ is the transverse wave function, $k_{n}^{2}=E-(n \pi / a)^{2}$ is the transport energy, $a_{n}$ is the input parameter, and $b_{n}$ is the reflection amplitude. Similarly for region II, we have

$$
\Psi_{\mathrm{II}}=\sum_{n} \chi_{n}(y)\left(c_{n} e^{i k_{n} x}+d_{n} e^{-i k_{n} x}\right)
$$

For region III,

$$
\Psi_{\mathrm{III}}=\sum_{n} \chi_{n}(x)\left(e_{n} e^{i k_{n} y}+f_{n} e^{-i k_{n} y}\right)
$$

where $c_{n}$ and $e_{n}$ are transmission amplitudes and $d_{n}$ and $f_{n}$ are input parameters. The wave function in region IV is a combination of wave functions in regions I, II, and III. At the boundaries of the various regions, we match the wave functions and their derivatives and this gives the desired transmission coefficients with which the scattering wave functions Eqs. (4)-(6) are also determined. Finally, if we choose point $O$ as the origin [see Fig. 1], the scattering matrix $s_{1 \beta}$ is defined as ${ }^{5}$

$$
\begin{gathered}
s_{11}=b_{1}, \\
s_{12}=c_{1} e^{i k_{1} a}, \\
s_{13}=e_{1} e^{i k_{1} a},
\end{gathered}
$$

where in general $b_{1}, c_{1}$, and $e_{1}$ are complex and $k_{1} a$ is the additional phase for outgoing wave functions. ${ }^{5}$

If we expand the ac admittance in powers of the frequency $\omega$, the coefficient of the linear term is called emittance. As mentioned in the introduction, the emittance $E_{\alpha \beta}$ is obtained from the various partial density of states: ${ }^{6}$

$$
E_{\alpha \beta}=\left(\frac{d N_{\alpha \beta}}{d E}-D_{\alpha \beta}\right) .
$$

The first term in the emittance gives the ac response of the system to the external potential change, while the second term is from the internal potential change induced by the external perturbations. We compute $d N_{\alpha \beta} / d E$ by applying Eq. (1), with a three-point numerical derivative to compute $d s_{\alpha \beta} / d E$. On the other hand, the internal contribution $D_{\alpha \beta}$ is related to the local PDOS, and within the Thomas-Fermi linear screening model is given by ${ }^{6}$

$$
D_{\alpha \beta} \equiv \int d \mathbf{r}\left[\frac{d n(\alpha, \mathbf{r})}{d E}\right]\left[\frac{d n(\mathbf{r})}{d E}\right]^{-1}\left[\frac{d n(\mathbf{r}, \beta)}{d E}\right],
$$

here the injectivity is calculable from the LPDOS, it is also calculable from the scattering wave function which is what we did in our numerical analysis,

$$
\frac{d n(\mathbf{r}, \alpha)}{d E}=\sum_{\beta} \frac{d n_{\alpha \beta}(\mathbf{r})}{d E}=\frac{1}{h J}\left|\Psi_{\alpha}(\mathbf{r})\right|^{2},
$$

where $J$ is the incident flux and $\Psi_{\alpha}(\mathbf{r})$ is the scattering wave function for electrons coming from the probe $\alpha$. Similarly, the emissivity $d n(\beta, \mathbf{r}) / d E$ equals the sum of LPDOS over its first index. In the absence of a magnetic field, the emissivity equals the injectivity. ${ }^{6}$ Finally, $d n(\mathbf{r}) / d E$ $=\Sigma_{\alpha} d n(\alpha, \mathbf{r}) / d E$ is the total local density of states.

Let us first analyze the ideal situation. If we sum over the index $\alpha$ for Eq. (9), we obtain

$$
\sum_{\alpha} D_{\alpha \beta}=\int d \mathbf{r}\left[\frac{d n(\mathbf{r}, \beta)}{d E}\right]=\sum_{\alpha} \int d \mathbf{r} \frac{d n_{\alpha \beta}(\mathbf{r})}{d E} .
$$

If Eq. (3) is precisely obeyed, we then immediately conclude that the electric current is conserved: $\Sigma_{\alpha} E_{\alpha \beta}=0$.

Our finite-size analysis of the GPDOS follows a quantity which measures the quality of the above current conservation: 

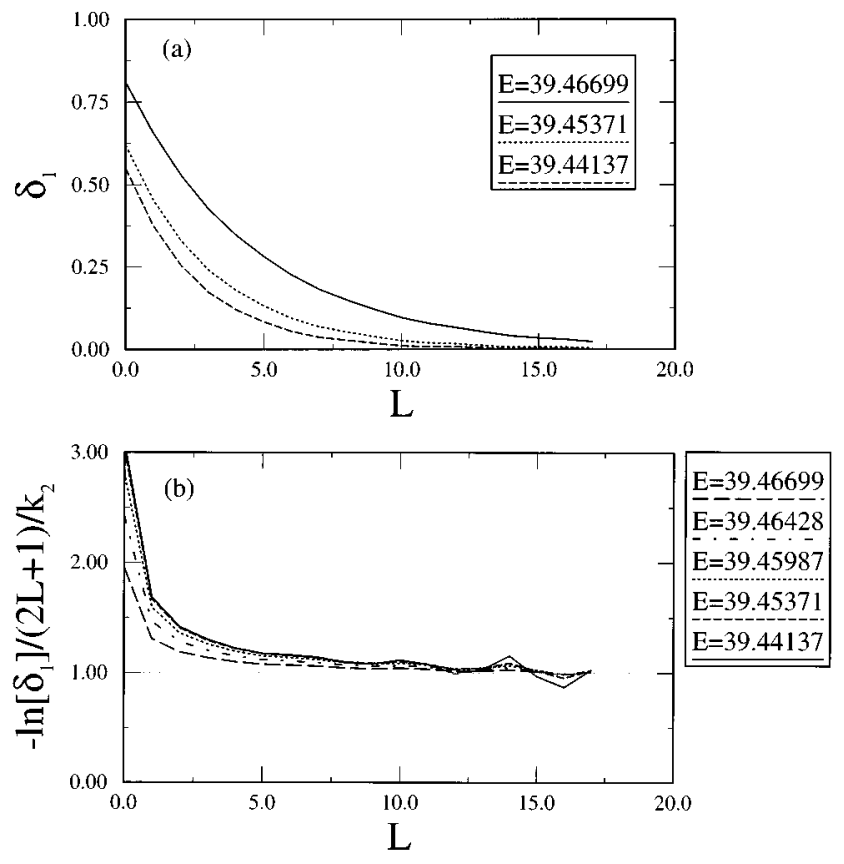

FIG. 2. (a). The difference, $\delta_{1}$ (in units of $2 m a^{2} / \hbar^{2}$ ), of the total PDOS as computed from the GPDOS and LPDOS from Eq. (12) as a function of the scattering volume linear size $L$. Solid line: at energy $E=39.466$ 99; dotted line: at energy $E=39.45371$; dashed line: at energy $E=39.44137$. (b) The quantity, $-\ln \left(\delta_{1}\right) /(2 L+1) / k_{2}$ as a function of the linear size $L$ for several incoming electron energies as shown. At large $L$, this quantity approaches unity, confirming the form of Eq. (13). Here (as well as in all other figures) the energy is in units of $\hbar^{2} / 2 m a^{2}$.

$$
\delta_{\beta}(L) \equiv \sum_{\alpha} E_{\alpha \beta}=\sum_{\alpha}\left(\frac{d N_{\alpha \beta}}{d E}-D_{\alpha \beta}\right),
$$

where $L$ is the scattering region size. Obviously $\delta_{\beta}=0$ if the current is precisely conserved.

Figure 2(a) shows $\delta_{1}(L)$ as a function $L$ for three energies close to the second subband edge which is located at $\mathcal{E}_{2}=39.4784$. A clear crossover to the large volume limit is revealed as $\delta_{1} \rightarrow 0$ when $L$ is increased. It is also clear that for energy closer to $\mathcal{E}_{2}$, the crossover is slower (solid line). We found that the decay of $\delta_{1}$ is essentially exponential for all energies examined, and has an interesting form for large $L$ :

$$
\delta_{1} \sim e^{-k_{2}(2 L+1)},
$$

where $(2 L+1)$ is precisely the scattering volume length from probe I to probe II, and $k_{2}$ is the momentum corresponding to the second subband energy $\mathcal{E}_{2}$. We have plotted $-\ln \left(\delta_{1}\right) /(2 L+1) / k_{2}$ in Fig. 2(b) for several energies. Our numerical data supports Eq. (13) quite well for large $L$, and for energies closer to $\mathcal{E}_{2}$. It is not difficult to understand the form of Eq. (13). Due to scattering at the junction where the three probes meet, complicated mode mixing takes place. While the incoming electron is in the first subband, mode mixing generates wave functions for many higher subbands, including the second subband, which become evanescent in the probes. For a scattering volume with a small $L$, the evanescent mode may "leak" out of the volume. However
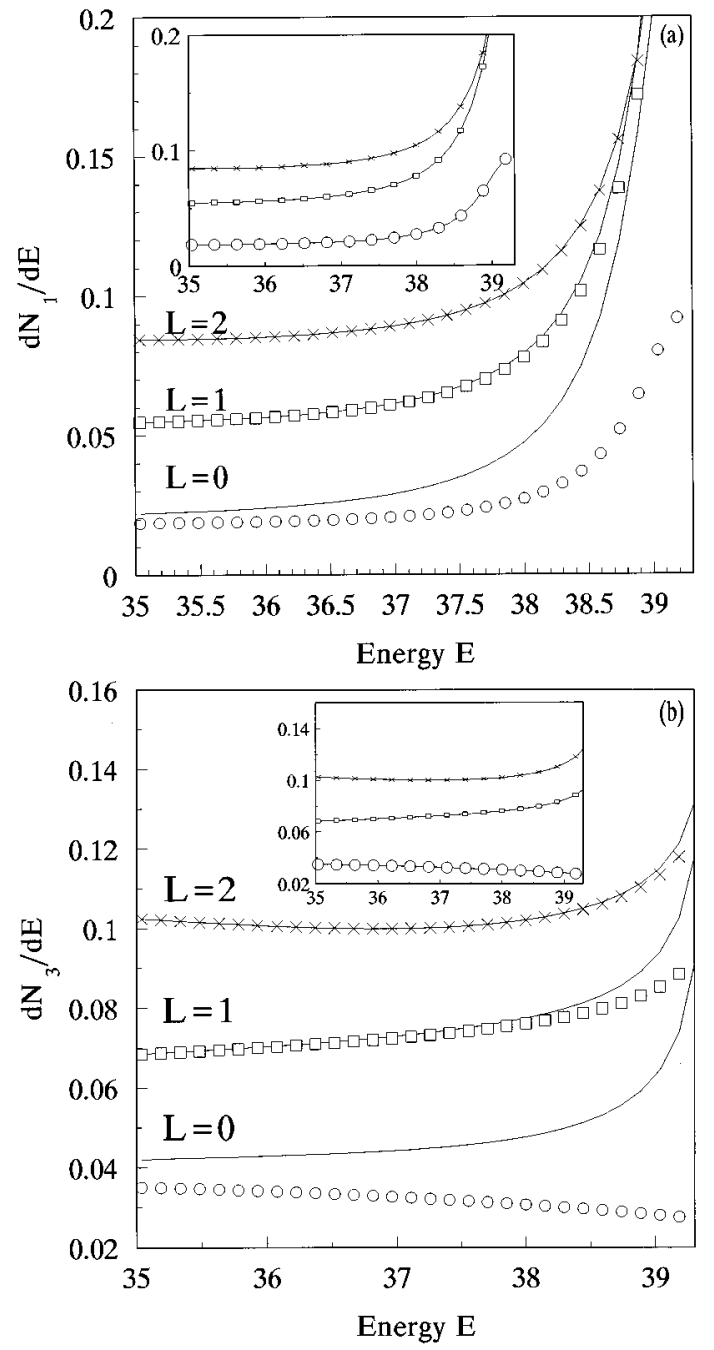

FIG. 3. Comparison of the total PDOS computed from the GPDOS and LPDOS, as a function of the incoming electron energy for three different sizes $L=0,1$, and 2 in the transmissive regime. (a) Electrons come from probe 1. (b) Electrons come from probe 3. The agreement of the total PDOS is good up to the "critical region" near the onset of the second subband. Insets: corresponding results for the total PDOS computed from LPDOS and the locally evaluated GPDOS of Eq. (15): perfect agreement is now obtained. The unit of PDOS is $2 m a^{2} / \hbar^{2}$

when we calculate the GPDOS from the scattering matrix, these "leaked" evanescent modes are not explicitly included, leading to a finite $\delta_{\beta}$. As we increase $L$, the evanescent modes decays away, and $\delta_{\beta}$ is reduced. In the simple model which can be solved exactly, ${ }^{4}$ a similar form to Eq. (13) was derived which was needed to correct the GPDOS in order to satisfy the precise current conservation. Our numerical study presented here reinforces the results of Ref. 4.

To further investigate the finite-size effect to GPDOS, in Figs. 3(a), (b) we plot the total DOS as obtained by GPDOS and LPDOS as functions of energy, for three system sizes $L$. The current conservation condition is satisfied very well for most of the first subband energies. When approaching the end of first subband, the current conservation condition is violated gradually, i.e., $\delta_{\beta} \neq 0$. We see that for the smallest scattering region $L=0$, the agreement of the two total DOS is at best reasonable when the incident electron is from probe 
I and is away from the second subband edge [Fig. 3(a)], and is quite bad when the electron is coming from probe III [Fig. $3(b)]$. The situation improves considerably when we increased the system size. As shown in Fig. 3, for $L=1$ and $L=2$, the agreement of the two total DOS are much better. However there is always a divergent behavior near the second subband for all sizes examined if the energy is made close enough to $\mathcal{E}_{2}$. Hence this is an intrinsic problem which goes away only when $L \rightarrow \infty$. The effect of increasing a finite size of the scattering volume is to decrease the "critical region" where the two total DOS disagree.

\section{A LOCAL APPROACH TO THE GPDOS}

To solve the finite-size problem, namely the problem of $\delta_{1}(L) \neq 0$, we emphasis that the origin of this difficulty is related to the mode mixing of the $2 \mathrm{D}$ scattering. This mode mixing is absent in 1D. Due to mode mixing there will be evanescent modes which "leak" out of the scattering region. However when we calculate the GPDOS from the scattering matrix using Eq. (1), these "leaked" evanescent modes are not explicitly included. On the other hand, when we compute the LPDOS internally using the scattering wave functions from Eq. (10), all the modes, including the evanescent modes, are included. Indeed, as emphasized by Büttiker, ${ }^{7}$ the ac transport formalism guarantees electric current conservation when the scattering volume is large enough to ensure that there is no electric field lines penetrating the surface of the scattering volume. This condition is certainly violated due to the "leaked" evanescent modes when the volume is small.

To proceed, we note that since evanescent modes do not contribute to electric current (but does to the DOS), it seems to be natural to use the conservation law to eliminate the need of computing GPDOS externally. This will be our approach. Hence, instead of computing GPDOS from Eq. (1), we shall instead use Eq. (3) for this purpose. The right hand side of (3) is entirely a local evaluation within the finite scattering region, thus can be computed accurately. With this in mind, the necessary next step is to evaluate the functional derivative of the scattering matrix with respect to the scattering potential landscape, as expressed in Eq. (2). In general this is the most difficult part, and it is rarely solvable analytically except in very special cases. ${ }^{4}$ For $1 \mathrm{D}$ systems, this functional derivative can be obtained via the Fisher-Lee ${ }^{8}$ relation, but this approach is not extendable to 2D. In the Appendix we give the 1D approach and outline the difficulty for $2 \mathrm{D}$ applications.

In order to compute the GPDOS locally using Eq. (3), we device the following numerical approach. We make use of a mathematical identity

$$
\frac{d \mathbf{s}_{\alpha \beta}}{d V}=\int d^{3} r \frac{\delta \mathbf{s}_{\alpha \beta}}{\delta U(\mathbf{r})},
$$

where $d \mathbf{s}_{\alpha \beta} / d V$ is calculated as follows: we add a constant potential $V$ in the scattering volume and compute the scattering matrix formally to get $\mathbf{s}_{\alpha \beta}=\mathbf{s}_{\alpha \beta}(V)$, then take the derivative and set $V=0$. Numerically the derivative can be easily carried out using finite differencing and we used a threepoint formula. Using Eqs. (3), (14), and (2), the GPDOS is

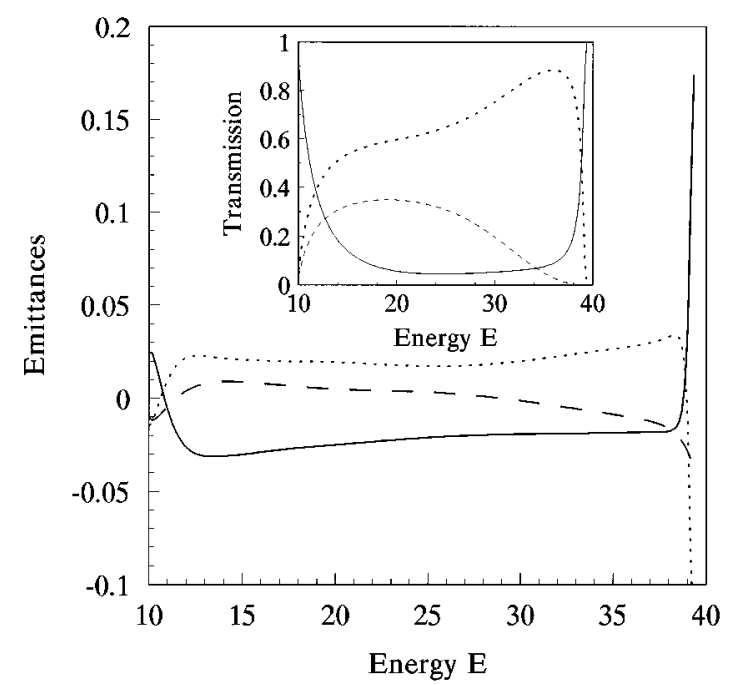

FIG. 4. The transmission coefficients and the emittance $E_{\alpha \beta}$ as functions of the incoming electron energy without the tunneling barriers. Solid line: $E_{11}$; dotted line: $E_{21}$; dashed line: $E_{31}$. Inset: solid line is reflection coefficient $R_{11}$; dotted line is transmission coefficient $T_{21}$; dashed line is $T_{31}$. The unit of emittance is $2 m a^{2} / \hbar^{2}$.

completely expressed by local quantities determined inside the scattering volume thus can be computed accurately for any system sizes,

$$
\frac{d N_{\alpha \beta}}{d E}=-\frac{1}{4 \pi i} \int d E\left(-\frac{d f}{d E}\right) \operatorname{Tr}\left[\mathbf{s}_{\alpha \beta}^{\dagger} \frac{d \mathbf{s}_{\alpha \beta}}{e d V}-\frac{d \mathbf{s}_{\alpha \beta}^{\dagger}}{e d V} \mathbf{s}_{\alpha \beta}\right]
$$

In this equation we have restored the Fermi function derivative $d f / d E$ for cases involving a nonzero temperature. We comment that this equation is valid in $1 \mathrm{D}$ as well.

\section{THE EMITTANCE}

Using result (15), we have recomputed the total DOS as obtained from the GPDOS and LPDOS. The two total DOS is plotted in the insets of Fig. 3. They now agree perfectly thus the finite-size problem for the emittance is solved. In the following we present the numerical results for the emittance obtained using Eqs. (15) and (9) which guarantee the current conservation.

In all the following results we have set the temperature to zero. Figure 4 shows the transmission coefficients (inset) and the emittance $E_{\alpha \beta}$ in the transmissive regime as a function of the incoming electron energy. In this case the system does not show resonance behavior and the transmission coefficients $T_{\alpha \beta}(E)$ are quite large for most of the energy range while the reflection coefficient $R_{11}$ is small [inset of Fig. 4]. It is interesting to find that the shape of the emittance is similar to that of the corresponding transmission coefficients. This is different from cases where quantum resonances dominate the transport (see below) and for that case the ac responses follow the dc transmissions only at the resonances. There are two different responses to the external time varying potential: capacitivelike and inductivelike depending on the sign of the emittance matrix element $E_{11}$. According to Eq. (8), $E_{11}$ consists of two terms: $d N_{11} / d E$ the capacitive- 


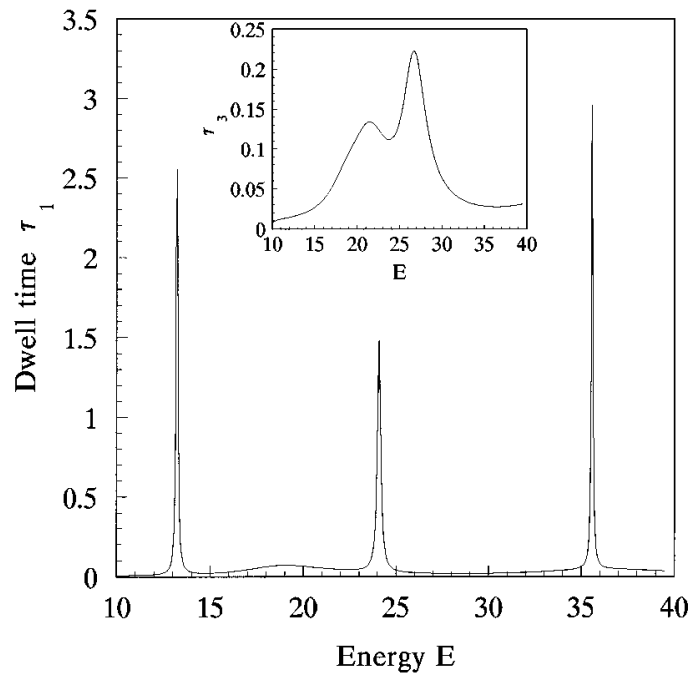

FIG. 5. Electron dwell time $\tau_{1}$ (in units of $4 \pi m a^{2} / \hbar$ ) as a function of the incoming electron energy in the tunneling regime. The three peaks indicate three resonance states in the system in this energy range. Inset: $\tau_{3}$.

like term and $D_{11}$ the inductivelike term. For a two-probe capacitor there is no dc current so that $d N_{12} / d E=0$. As a result $E_{12}$ is negative. Therefore for a capacitor $E_{11}=-E_{12}$ is positive. Extending this notion, one concludes that the system responds capacitively if $E_{11}$ is positive. For a ballistic conductor with complete transmission $d N_{11} / d E$ vanishes and $E_{11}$ is negative. In other words, negative $E_{11}$ gives an inductivelike response. These different responses are clearly shown in Fig. 4.

The ac transport properties are very different in the tunneling regime. To establish such a regime, we have put tunneling barriers inside probes 1 and 2 at the junctions between the probes and the scattering volume. In particular the barrier heights are $V_{\text {barrier }}=40 E_{1}$, and the width is 0.1 where the width of the wire $a$ has been set to one. No barrier is added in probe 3 . We have also included a potential well with depth $V_{\text {well }}=-40 E_{1}$ in the center of the scattering volume with a size of $2.8 \times 1.9$. The well and barriers establish several transport resonances, these are clearly marked by the sharp peaks in the electron dwell time defined as ${ }^{9}$

$$
\tau_{\alpha}=\frac{1}{J} \int_{\Omega}\left|\Psi_{\alpha}(\mathbf{r})\right|^{2} d^{3} \mathbf{r}
$$

where $\Omega$ is the scattering volume. $\tau_{1}$ is plotted against energy in Fig. 5 while the inset shows $\tau_{3}$. The dwell time measures the duration an electron spends in the scattering volume. Thus if transport is mediated by resonance states we expect much longer dwell times ${ }^{10}$ at the resonances. This idea has recently been proved by Iannaconne. ${ }^{11}$ Figure 5 shows that three resonance states, with energies $E_{1}=13.2$, $E_{2}=24.1$, and $E_{3}=35.6$ are established. The quantum resonances also leads to sharp peaks in the transmission coefficient $T_{21}$ and reflection coefficient $R_{11}$, as shown by the solid lines of Figs. 6(a), 6(b). At these resonances both the GPDOS and LPDOS take maximum values, leading to the sharp jumps in the emittance $E_{11}$ and $E_{21}$ as shown by the dotted lines in Fig. 6. The variations of $E_{11}$ and $E_{21}$ as func-
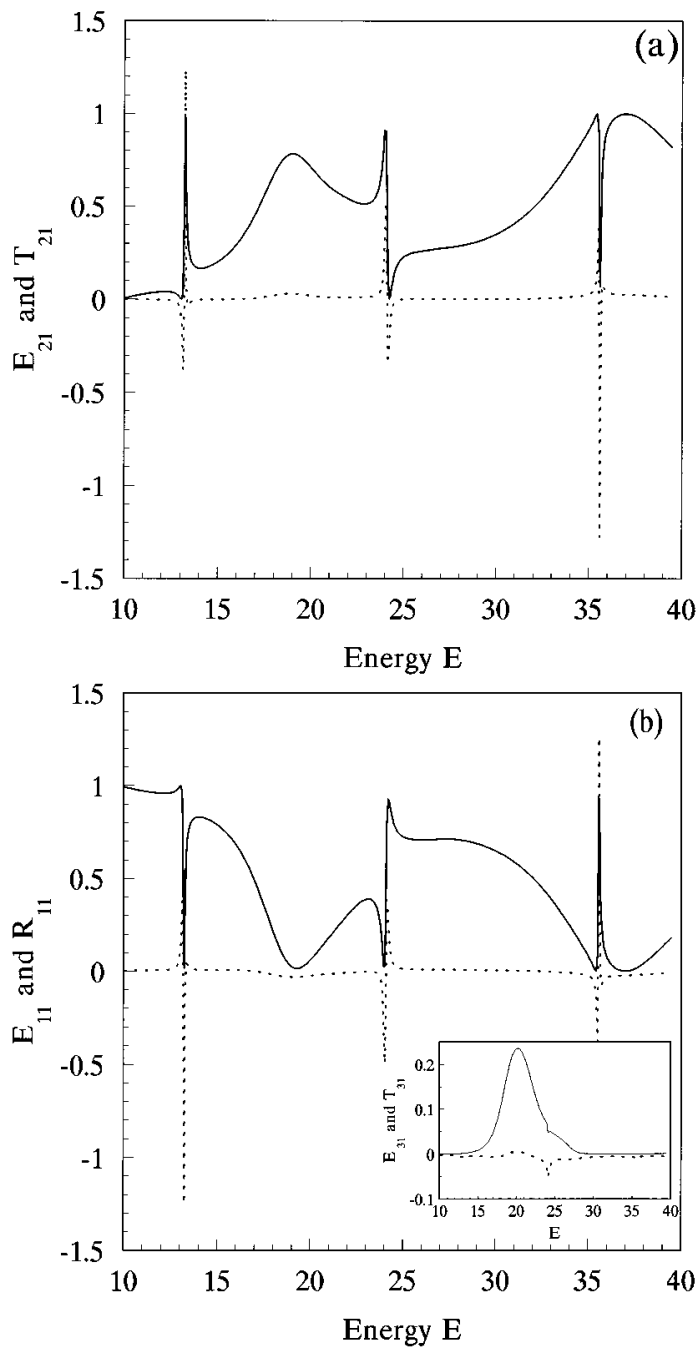

FIG. 6. The transmission coefficients and the emittance $E_{\alpha \beta}$ as functions of the incoming electron energy in the tunneling regime. (a) Solid line: $T_{21}$; dotted line: $E_{21}$. (b) Solid line: $R_{11}$; dotted line: $E_{11}$. Inset of (b): Solid line: $T_{31}$; dotted line: $E_{31}$.

tions of energy $E$ are very closely correlated with those of $R_{11}(E)$ and $T_{21}(E)$ near the resonances. Since there is no tunneling barrier in probe 3 , the resonance transmission to that probe is not as sharp, and the transport behavior shows a mixture of tunneling and transmission as shown in the inset of Fig. 6(b).

In the tunneling regime the ac response changes sharply from inductivelike behavior at one side of the resonance energy to capacitivelike on the other side of the resonance or vice versa, in distinctive difference as compared to the transmissive case discussed above. Let us examine $E_{11}$ near resonance $E_{3}$. As the energy approaches $E_{3}$, the system first responds inductively and is followed by a strong capacitive response. This behavior is clearly related to the fact that the resonance is characterized by a complete reflection indicated by the large peak in the reflection coefficient [see Fig. 6(b)]. This behavior has been seen previously in 2D quantum wires. ${ }^{4}$ On the other hand, for $1 \mathrm{D}$ resonance tunneling, a Breit-Wigner-type transmission resonance gives rise to an ac response $^{7}$ similar to that discussed here. When the incident energy is near the resonance $E_{1}$, the ac response is reversed: 
first capacitivelike and then inductivelike. Hence the behavior near $E_{1}$ and $E_{3}$ are very different. For an energy near $E_{1}$ the emittance behaves like an odd function but near $E_{3}$ it is like an even function. The reason, as we have checked numerically, is that the external and the internal responses do (not) reach the maximum at the same energy for $E$ near $E_{3}$ $\left(E_{1}\right)$. This behavior of $E_{11}$ is also a manifestation of the reflection coefficient $R_{11}$. As the energy sweeps through $E_{1}$, the strong capacitivelike ac response is due to the complete reflection peak, and the following inductivelike response is because the reflection coefficient $R_{11} \approx 0$. Hence in the ac response of a system, near a quantum resonance whether it is voltage following current (capacitive) first, or current following voltage (inductive) first, can only be determined by detailed analysis and the outcome depends on the peculiarities of the system such as the existence of a third probe as we have studied here. In the inset of Fig. 6(b) we show the emittance matrix elements $E_{13}$. Although they have much smaller values they do exhibit dips around three resonant energies $E_{1}, E_{2}$, and $E_{3}$.

\section{SUMMARY}

In summary, to obtain precise results of the lowfrequency emittance from numerical analysis of $2 \mathrm{D}$ or $3 \mathrm{D}$ mesoscopic conductors, we need to solve the problem associated with the finite-size effect of the scattering region. This work identifies the origin of the problem as due to the localized modes which were not included in the evaluation of the global partial density of states. Using a quite typical multiprobe system which is a three-probe $2 \mathrm{D}$ quantum wire, we have demonstrated where and how the finite-size effect shows up. The finite-size effect leads to current nonconservation. Increasing the scattering region size does not solve the problem, it only shrinks the nonconservation regime. The problem goes away only when the system size is infinitely large.

To solve the finite-size problem, we proposed a numerical procedure and a formula for computing the global partial density of states which is precise for any finite scattering volume of a quantum conductor. As GPDOS plays an important role in the ac transport theory, our result provides a useful tool for further numerical investigations of the dynamic admittance. In this formulation the electric current conservation is satisfied automatically. Finally, we mention that this formulation of computing the GPDOS locally also applies to the investigation of nonlinear dc transport. ${ }^{12}$ Applying the procedure to the three-probe conductor, the divergences of the emittance at each subband edge are removed. This allows us to present precise results concerning the behavior of the emittance matrix.

\section{ACKNOWLEDGMENTS}

We gratefully acknowledge support by a RGC grant from the Government of Hong Kong under Grant No. HKU 261/95P, a research grant from the Croucher Foundation, the Natural Sciences and Engineering Research Council of Canada, and le Fonds pour la Formation de Chercheurs et l'Aide à la Recherche de la Province du Québec. We thank the Computer Center of the University of Hong Kong for computational facilities and the access of SP2 supercomputer.

\section{APPENDIX}

In this appendix, we first outline how to obtain LPDOS $d n_{\alpha \beta} / d E$ for $1 \mathrm{D}$ systems following Ref. 1 . We then demonstrate that this $1 \mathrm{D}$ method is not easily generalizable to $2 \mathrm{D}$.

For an 1D conductor, one makes use of the Fisher-Lee relation ${ }^{8}$ between the scattering matrix and the retarded Green's function:

$$
s_{\alpha \beta}=-\delta_{\alpha \beta}+i \hbar \sqrt{v_{\alpha} v_{\beta}} G\left(x_{\alpha}, x_{\beta}\right),
$$

where $x_{\alpha}$ is the boundary of the scattering region. The functional derivative of the Green's function $\delta G / \delta U$ is given by ${ }^{1}$

$$
\frac{\delta G\left(x_{\alpha}, x_{\beta}\right)}{\delta U(x)}=G\left(x_{\alpha}, x\right) G\left(x, x_{\beta}\right) .
$$

Furthermore one can prove that for 1D systems the following relation is true: ${ }^{1}$

$$
G\left(x_{1}, x\right) G\left(x, x_{2}\right)=G\left(x_{1}, x_{2}\right) G(x, x)
$$

for $x_{1}<x<x_{2}$. Using Eqs. (A1)-(A3), it is not difficult to derive, ${ }^{1}$ for $1 \mathrm{D}$ systems, the following expressions for the LPDOS,

$$
\frac{d n_{\alpha \beta}(\mathbf{r})}{d E}=\frac{1}{2} T_{\alpha \beta} \frac{d n(\mathbf{r})}{d E},
$$

for $\alpha \neq \beta$ and

$$
\frac{d n_{\beta \beta}(\mathbf{r})}{d E}=\frac{d n(\mathbf{r}, \beta)}{d E}-\frac{1}{2} \sum_{\alpha \neq \beta} T_{\alpha \beta} \frac{d n(\mathbf{r})}{d E},
$$

where $T_{\alpha \beta}$ is the transmission coefficient from lead $\beta$ to $\alpha$. In these results, $d n(\mathbf{r}, \beta) / d E$ is defined as

$$
\frac{d n(\mathbf{r}, \beta)}{d E}=\sum_{\alpha} \frac{d n_{\alpha \beta}(\mathbf{r})}{d E},
$$

which is the injectivity introduced in Sec. II and it measures the additional local charge density brought into the sample at point $\mathbf{r}$ by the oscillating chemical potential at probe $\beta$. From Eq. (10) the injectivity can be expressed in terms of the scattering wave function. ${ }^{6}$ After obtaining the total local $\operatorname{DOS} d n / d E$ from summing up the injectivity index $\beta$, one is able to calculate the LPDOS via Eqs. (A4) and (A5).

For 2D systems which is the interest here, the Fisher-Lee relation has the form ${ }^{5}$

$$
\begin{aligned}
s_{\alpha n \beta m}= & -\delta_{\alpha n \beta m}+i \hbar \sqrt{v_{\alpha n} v_{\beta m}} \iint G\left(x_{\alpha}, y_{\alpha}, x_{\beta}, y_{\beta}\right) \\
& \times \chi_{\alpha n}\left(y_{\alpha}\right) \chi_{\beta m}\left(y_{\beta}\right) d y_{\alpha} d y_{\beta},
\end{aligned}
$$

where $\chi_{\alpha n}$ is the transverse wave function in lead $\alpha$. In 2D, the equations similar to Eqs. (A4) and (A5) do not seem to apply. This is because Eq. (A3) does not hold in 2D. We can see that clearly by considering a simple system: a pipe with a $\delta$-function potential $V(\mathbf{r})=\gamma \delta\left(\mathbf{r}-\mathbf{r}_{0}\right)$. This system has mode mixing which is essential for a $2 \mathrm{D}$ system and is ex- 
actly solvable. ${ }^{4}$ The Green's function $G\left(\mathbf{r}, \mathbf{r}^{\prime}\right)$ for this case can be derived analytically and is given by

$$
\begin{aligned}
G\left(\mathbf{r}, \mathbf{r}^{\prime}\right)= & G_{0}\left(\mathbf{r}, \mathbf{r}^{\prime}\right)+\gamma G_{0}\left(\mathbf{r}, \mathbf{r}_{0}\right) \\
& \times G_{0}\left(\mathbf{r}_{0}, \mathbf{r}^{\prime}\right) /\left[1-\gamma G_{0}\left(\mathbf{r}_{0}, \mathbf{r}_{0}\right)\right],
\end{aligned}
$$

where $G_{0}$ is the Green's function for a pipe with transverse wave function $\chi_{m}$. From Ref. 5, we have $G_{0}\left(\mathbf{r}, \mathbf{r}^{\prime}\right)$ $=\Sigma_{m}\left(-i / v_{m}\right) \chi_{m}(y) \chi_{m}\left(y^{\prime}\right) \exp \left(i k_{m}\left|x-x^{\prime}\right|\right)$. In this case, one sees that Eq. (A3) is not satisfied because of the mode mixing. Hence the 1D procedure outlined in the last paragraph to obtain LPDOS is not applicable in 2D, in general.
Interestingly, we found that if the incoming electron energy is restricted to the first subband, Eqs. (A4) and (A5) do hold in 2D. For a pipe with a $\delta$ function scatterer this can be shown analytically. For a T-shaped 2D scattering junction we have verified it numerically. However since this is only a very special case for $2 \mathrm{D}$ conductors, it is not general as we have explicitly checked numerically that these equations do not hold when there is more than one transport subbands involved in the scattering process. We have also checked numerically that the semiclassical expression in 2D for the LPDOS (Ref. 12) is not accurate. Hence a general procedure discussed in this paper is necessary.
${ }^{1}$ V. Gasparian, T. Christen, and M. Büttiker, Phys. Rev. A 54, 4022 (1996).

${ }^{2}$ For the nonequilibrium Green's function approach see, for example, N. S. Wingreen, A. Jauho, and Y. Meir, Phys. Rev. B 48, 487 (1993); T. K. Ng, Phys. Rev. Lett. 76, 487 (1996); T. Ivanov, J. Phys. Condens. Matter 8, 3427 (1996).

${ }^{3}$ Y. Avishai and Y. B. Band, Phys. Rev. B 32, 2674 (1985).

${ }^{4}$ Jian Wang, Qingrong Zheng, and Hong Guo, Phys. Rev. B 55, 9770 (1997).

${ }^{5}$ S. Datta, Electronic Transport in Mesoscopic Conductors (Cambridge University Press, Cambridge, England, 1995).

${ }^{6}$ T. Christen and M. Büttiker, Phys. Rev. Lett. 77, 143 (1996); M. Büttiker and T. Christen, in Quantum Transport in Semiconduc- tor Submicron Structures, edited by B. Kramer (Kluwer, Netherlands, 1996), p. 263; Dynamic and Nonlinear Transport in Mesoscopic Structures, in Theory of Transport Properties of Semiconductor Nanostructures, edited by E. Schöll (Chapman and Hall, London, 1996).

${ }^{7}$ M. Büttiker, H. Thomas, and A. Prêtre, Z. Phys. B 94, 133 (1994).

${ }^{8}$ D. S. Fisher and P. A. Lee, Phys. Rev. B 23, 6851 (1981).

${ }^{9}$ M. Büttiker, Phys. Rev. B 27, 6178 (1983).

${ }^{10}$ Jian Wang, Yongjiang Wang, and Hong Guo, Appl. Phys. Lett. 65, 1793 (1994).

${ }^{11}$ G. Iannaccone, Phys. Rev. B 51, 727 (1995).

${ }^{12}$ T. Christen and M. Büttiker, Europhys. Lett. 35, 523 (1996). 